\title{
El modelo de aprendizaje alostérico de Andre Giordan y su aplicación a la capacitación de adultos.
}

\author{
The allosteric learning model of Andre Giordan and its application to the adult training.
}

Ana Jesus Aguilar Angeletti 1,a

\section{RESUMEN}

Aspectos relevantes de la investigación realizada por la autora, titulada "Una aproximación del Modelo Alostérico de aprendizaje a la capacitación de adultos: análisis cualitativo de las concepciones distorsionadas sobre educación sexual de profesores de educación secundaria”, son presentados, analizados y evaluados, concluyendo que el Modelo Alostérico de Aprendizaje propuesto por André Giordan para el proceso enseñanza-aprendizaje de los niños, puede ser exitosamente aplicado en adultos. Antecedentes y características del modelo son descritos, destacando su utilidad para identificar las distorsiones en la capacitación de los adultos, orientándolos hacia la aplicación de mecanismos de auto-transformación para corregirlas. Se enfatiza en la importancia de su uso en temas de Salud Pública, tales como los relacionados con la Educación Sexual en la escuela, siendo necesario contar con profesores con cien por ciento de las concepciones correctas con el fin de evitar el efecto multiplicador de sus distorsiones en los estudiantes.

PALABRAS CLAVE: Modelo de Aprendizaje Alostérico, enseñanza, aprendizaje, capacitación de adultos.

\section{SUMMARY}

Relevant aspects of the research done by the author entitled "An approximation of Allosteric Learning Model for the Adult Training: qualitative analysis of distorted conceptions about Sexual Education in teachers of Secondary Education" are presented, analyzed and evaluated, concluding that the Allosteric Learning Model proposed by André Giordan to the teaching-learning process of children, can be successfully applied in adults. Background and characteristics of the model are described, highlighting its utility to identify distortions in adult training, guiding them towards the implementation of self-transformation mechanisms in order to correct them. It emphasizes the importance of its use in Public Health issues, such as those related to Sexual Education in school, being necessary to have teachers with one hundred percent correct conceptions to avoid the multiplier effects of their distortions on the students.

KEYWORDS: Allosteric Learning Model, teaching, learning, adult training.

\section{INTRODUCCIÓN}

El proceso enseñanza-aprendizaje, en todo esfuerzo educativo, ha ido evolucionando a partir de la aplicación de diversos modelos explicativos. En lo que concierne al aprendizaje, los modelos de mayor uso hacen referencia a los aspectos evolutivos relacionados con el pensamiento de Piaget (1926); otros, se basan en la corriente psicopedagógica no directiva de Rogers; o parten de las teorías conductuales como las de Skinner, Keller, y Bijou (Aguilar, 1997).

Facultad de Psicología Leopoldo Chiappo Galli de la Universidad Peruana Cayetano Heredia. Lima, Perú.

a Profesora Principal. 
En las últimas décadas se han considerado también teorías cognitivas como las de Ausubel (1982), Gagne (1979), Bandura (1983) sobre el aprendizaje social, o las teorías constructivistas en las que destacan también los aportes de Piaget así como los de Vigotsky (1978), entre otras.

Dada la amplitud y complejidad del campo cognitivo del ser humano, las investigaciones sobre el tema continuaron enriqueciéndose con el constructivismo y nuevos análisis aparecieron principalmente en relación al aprendizaje, como el Modelo Alostérico de Aprendizaje de André Giordán (1989).

En un intento por sistematizar los datos y características de los modelos explicativos del aprendizaje de más frecuente uso en nuestro medio, se presenta una breve descripción de las características más relevantes de tres de ellos:

1. Modelo del Refuerzo de B.F. Skinner.

2. Modelo Cognoscitivo de David Ausubel.

3. Modelo Jerárquico de Robert Gagné.

\section{Modelo del Refuerzo de B. F. Skinner}

Se basa en el uso del refuerzo como factor fundamental del aprendizaje; considerando que para adquirir, incrementar o extinguir un aprendizaje, se debe hacer contingente (de modo inmediato) una consecuencia ante las ejecuciones del sujeto.

El enfoque de Skinner (1979) parte del análisis de los datos empíricos para llegar a la generalización; rechaza cualquier método que no se base en la observación sensorial; se limita a describir antes que a explicar y exige del investigador que los datos observados debe identificarlos fácilmente, con claridad y sin ambigüedad. Razón por la que se le considera como radical con un esquema donde sólo incorpora el estímulo, la respuesta y el refuerzo, sin incluir las variables internas del que aprende, en cuanto a los procesos mediadores como las motivaciones, actitudes, pensamientos, valores, expectativas, etc.

Identifica dos tipos de aprendizaje: el respondiente, de naturaleza refleja, y el operante o instrumental, relacionado con las ejecuciones que aprende el sujeto. Clasifica al refuerzo como primario - estímulo que sin historia previa refuerza al comportamiento dado cierto estado de deprivación, el alimento por ejemplo - o como secundario - estímulo que refuerza el comportamiento en base a una historia previa, el dinero, los elogios, los premios, por ejemplo.
También clasifica al refuerzo como positivo o como negativo. El refuerzo positivo incrementa la frecuencia y/o duración del comportamiento, como el felicitar al grupo por haber aprendido a utilizar un programa estadístico para procesar datos epidemiológicos sobre la tuberculosis. El refuerzo negativo, reduce o extingue la frecuencia y/o duración del comportamiento, como cerrar la puerta de ingreso al aula a los alumnos que llegan tarde a la charla de capacitación.

Asimismo, el enfoque de Skinner plantea que la manera cómo son presentados los refuerzos en una situación de aprendizaje, responde a un programa específico que se adapta y adecúa a las condiciones individuales y del medio; razón por la que clasifica los programas según sean o no intermitentes o según el número de ejecuciones (de razón) o según su duración (de intervalo).

En cuanto a la aplicación del enfoque del refuerzo, se considera que es muy útil para la elaboración de textos programados y de los diseños de instrucción; en el empleo de máquinas de enseñanza, de juegos educativos; en el empleo de Técnicas de Modificación de Conducta; y, en casos de Niños con Necesidades Educativas Especiales.

\section{Modelo cognoscitivo de David Ausubel}

$\mathrm{Su}$ interés fundamental es el aprendizaje significativo y simbólico. Hace referencia al aprendizaje dirigido, en el que el contenido total de lo que se va a aprender se presenta al sujeto en su forma final, para que lo internalice y posteriormente lo reproduzca; diferenciándolo del aprendizaje por descubrimiento en el que el contenido debe ser elaborado por el sujeto, cuya tarea es descubrir algo para luego hacerlo significativo.

Ausubel (1982) destaca la intervención en el aprendizaje tanto de factores internos, como externos del sujeto. Considera que son factores internos: su estructura cognoscitiva, disposición del desarrollo, capacidad intelectual, motivación, actitudes, personalidad y experiencias anteriores.

Como factores externos describe la frecuencia y condiciones generales del aprendizaje; la organización y arreglo de los materiales de enseñanza; los factores sociales y de grupo; las características del profesor, como su capacidad intelectual, su preparación y conocimientos sobre la materia, su experiencia y competencia pedagógica, su flexibilidad y 
comunicación, su trato correcto, agradable y sencillo, así como su capacidad de innovación.

Al incorporar en su análisis eventos internos y externos, se le ubica en el esquema metodológico que considera no sólo lo observable directamente, sino también aquellas variables internas de tipo cognitivo y simbólicas, enfatizando en los aprendizajes de representaciones que consisten en adquirir símbolos (su significado) generalmente de palabras; de proposiciones, mediante los cuales se adquieren significados de grupos de palabras combinadas en proposiciones u oraciones; y de conceptos, que consisten en adquirir ideas genéricas categoriales de conjunto de proposiciones.

El modelo cognoscitivo de David Ausubel se aplica con mayor frecuencia en el diseño curricular, la educación superior, la capacitación de adultos y la educación de genios o superdotados.

\section{Modelo jerárquico de Robert Gagné}

Al igual que el modelo anterior se le ubica en el esquema metodológico pues estudia tanto variables internas como externas del aprendizaje. Identifica condiciones internas y externas, plantea que cada aprendizaje parte de una capacidad distinta interna.

Gagné (1979), desde una perspectiva jerárquica, clasifica hasta ocho tipos de aprendizaje:

$1^{\circ}$ Aprendizaje respondiente: reacción ante una señal.

$2^{\circ} \mathrm{E}-\mathrm{R}$ : aprendizaje de respuesta precisa ante un estímulo discriminado.

$3^{\circ}$ Encadenamiento: Aprendizaje de cadena de conexiones $\mathrm{E}-\mathrm{R}$.

$4^{\circ}$ Asociación verbal: aprendizaje de cadenas verbales.

$5^{\circ}$ Discriminación: aprender características diferenciándolas entre sí.

$6^{0}$ Aprendizaje de conceptos: identifica clase de objetos o fenómenos.

$7^{\circ}$ Aprendizaje de principios: de cadena de conceptos.

$8^{\circ}$ Aprendizaje de resolución de problemas: combinación de principios para resolver situaciones, que requiere de amplio uso del razonamiento, la lógica y la abstracción.

El autor destaca que la formación de aprendizajes requiere de pre-requisitos, constituyéndose cada tipo de aprendizaje en pre-requisito del siguiente tipo, organizándose así una jerarquía ordenada de menor a mayor complejidad. Asimismo, destaca la importancia de la transferencia tanto horizontal como vertical; considerando que la horizontal posibilita la generalización de lo aprendido en un mismo tipo de aprendizaje a diferentes contextos y/o situaciones y que la transferencia vertical posibilita el pasaje de un tipo inferior a otro tipo superior de aprendizaje, según la jerarquía antes descrita.

Clasifica hasta cinco resultados o productos del aprendizaje:

1. Información Verbal:

Mediante los métodos para transmitir conocimientos, se adquieren unidades de información clasificables como hechos, nombres, conceptos, principios.

2. Habilidades intelectuales:

Aprender cómo hacer o transformar algo con destrezas que capacitan al aprendiz para actuar simbólicamente sobre la realidad, mediante la discriminación de semejanzas y diferencias, definición de conceptos, aplicación de reglas y principios explicativos.

3. Estrategias Cognoscitivas:

Los mecanismos cognoscitivos que guían la atención, el recuerdo y el pensamiento, empleados para pensar (reflexionar) sobre lo aprendido y que regulan internamente las ejecuciones del aprendiz en su contexto.

4. Actitudes:

Estado interno adquirido del aprendiz que influye sobre la elección de su acción personal hacia un objeto actitudinal (persona, hecho o cosa), que está asociado a sus valores y que afecta su actuación.

5. Habilidades motrices:

Relacionadas con las destrezas que intervienen en actividades motoras organizadas y que conducen a ejecutar movimientos corporales motores finos o gruesos.

El modelo jerárquico de Robert Gagné se aplica en el proceso de planificación educativa, en el diseño de las secuencias instruccionales del proceso enseñanzaaprendizaje que incorpora aspectos vinculados con los pre-requisitos y la transferencia. Asimismo, el modelo tiene estrecha relación con el enfoque sistémico aplicado a la educación.

En la búsqueda de nuevas alternativas que enriquezcan todo esfuerzo de enseñanza-aprendizaje, se identificó el modelo propuesto por André Giordán (1995). 


\section{EL MODELO ALOSTÉRICO DE APRENDIZAJE DE ANDRÉ GIORDÁN (MAADAG)}

\section{Origen del término "alostérico"}

El término "alostérico" procede de la Bioquímica y la Biología Molecular. Según los textos de Bioquímica de Lehninger (1993) y de Harper (1994), "alostérico" proviene de los vocablos griegos (allos = otros) $\mathrm{y}$ $($ steros $=$ espacio $)$.

El modelo alostérico fue propuesto en 1965 por Jacques Lucien Monod (Monod, Wyman y Chageux, 1995), el mismo año que le otorgaban el Premio Nóbel de Fisiología y Medicina por haber postulado la existencia del RNA Mensajero en 1961.

Monod en 1993 publica su celebrado ensayo sobre la filosofía natural de la biología moderna: "El azar y la necesidad". En esta obra señala la condición de "Relay Molecular" que exhiben las enzimas alostéricas y que a continuación se comenta brevemente por constituir el referente central y punto de partida del modelo de aprendizaje alostérico de André Giordán.

Todas las enzimas tienen un sitio activo, que cataliza una reacción en la substancia sobre la cual actúan. Las enzimas alostéricas, además de este sitio activo, tienen "otro" (allos), que regula la actividad de la enzima porque frena o acelera su actividad catalítica. Se postula que, a partir del sitio alostérico, se generan cambios conformacionales -como el movimiento de las fichas de dominó- que modifican la forma y por lo tanto la función del sitio activo.

\section{El MAAdAG propuesto por André Giordán.}

El Modelo Alostérico de Aprendizaje de André Giordán (MAAdAG) fue propuesto para explicar la progresión en la construcción del saber científico, partiendo de la caracterización de las concepciones de los niños, su origen y los procesos de cambio de las mismas. Enfatiza en la necesidad de modelos que prioricen la relación entre una estructura de pensamiento ya constituida y los nuevos elementos de conocimiento exteriores (Giordan, 1995).

Giordán señala que los métodos habituales de transmisión del saber y las diversas innovaciones pedagógicas no generan los resultados esperados, presentándose en los alumnos un determinado número de "errores" de razonamiento o de ideas "erróneas" y que las concepciones erróneas pueden presentarse hasta de tres formas diferentes: a) Antes del curso

Los alumnos previamente a la nueva enseñanza sobre un objeto de estudio, poseen un cierto número de concepciones erróneas que se pueden categorizar y describir con detalles.

b) Al finalizar el curso

El aprendizaje de los nuevos conocimientos concretos depende de las concepciones de los alumnos, que si son erróneas, la interpretación de la nueva información también será equivocada.

c) En lo que realmente había comprendido

Si la enseñanza no lo tiene en cuenta, las ideas existentes constituirán un obstáculo, conservándose las nociones enseñadas de modo deformado, "pegándose" o permaneciendo aisladas del saber anterior.

En consecuencia, para un profesor es sumamente importante explorar y conocer las concepciones de los alumnos, pues permite adaptar mejor la enseñanza y proponer estrategias más eficaces que tengan en cuenta la situación o contexto de la enseñanza, las intervenciones del profesor, el uso de las ayudas didácticas así como la planificación de las mismas.

Giordán considera que en el aprendizaje, el pensamiento del alumno no se presenta como un sistema de registro lineal y pasivo, ni funciona como una simple estructura de memorización. La estructura se constituye progresivamente a través de la enseñanza, de los medios de divulgación $\mathrm{y}$, principalmente, a través de las experiencias de vida cotidiana. Mediante ella se decodifica la información, dando sentido a las nuevas situaciones, insertándose y organizándose así los nuevos datos según reglas específicas y en estrecha relación con las concepciones ya existentes. Señala que para el éxito de la enseñanza, será necesario, por tanto, discriminar estas concepciones, sus fuentes y/o circunstancias en las que se adquirieron, cómo funcionan realmente e interferir con ellas (Giordan, 1995).

Enfatiza en que el problema central del aprendizaje no consistirá en el desglose de la materia a enseñar en unidades básicas de conocimientos, referida cada una a un ejercicio particular o en la simple decodificación de elementos verbales transformables en elementos abstractos; sino en considerar modelos orientados a relacionar la estructura de pensamiento ya constituida con los nuevos elementos de conocimiento que deberán se aprendidos. 
Giordan (1995) hace referencia a Ausubel y Novakal señalar que el aprendizaje de conocimientos supone una integración en una estructura cognitiva ya existente que facilita la memorización y constituye un punto de anclaje para nuevos datos; asimismo, comenta que según Novak, la integración es facilitada por "puentes cognitivos" que hacen significativa la información al relacionarla con la estructura global pre-existente.

En cuanto a la teoría de Piaget manifiesta que está basada en las ideas de "asimilación y acomodación" y que conducen a una "abstracción reflexiva"; es decir, el alumno introduce en su organización cognitiva personal los datos del mundo exterior, las nuevas informaciones las trata en función de las adquisiciones anteriormente constituidas, pudiendo originar la acomodación, transformando los esquemas de pensamiento existentes en función de las nuevas circunstancias. En este mismo sentido refiere a Bachelard quien plantea que ante un conocimiento científico, no está "vacía" la cabeza del alumno, sino que ya tiene cierta estructuración inicial de la experiencia. Sus ideas previas están en el punto de partida de procedimientos de elaboración de sus conceptos y si esos saberes previos son ignorados, sólo serán alejados temporalmente y no serán suprimidos (Giordan, 1995).

Giordán (1989) además refiere: "No obstante, es preciso avanzar hasta la actualidad, en donde nuestros trabajos de didáctica, confirmados en parte por estudios de psicología cognitiva, de inteligencia artificial y de epistemología, muestran que se conoce a la vez "gracias a" (Gagne), "a partir de" (Ausubel) y "con" (Piaget), nuestros conocimientos anteriores y, al mismo tiempo, se aprende "contra" (Bachelard) estos últimos.

Asume que la producción de aprendizajes se basa principalmente en dos aspectos:

- las condiciones que el alumno debe poner en marcha para generar la autotransformación de su estructura conceptual,

- la necesidad de un entorno didáctico adecuado, indispensable para favorecer ese cambio.

Sin embargo, comenta que sobre estos dos aspectos se comprueba que las principales teorías psicológicas o epistemológicas aún no plantean su total solución. Para formular el esbozo del modelo que ha denominado modelo alostérico de aprendizaje, plantea analogías entre los procesos de aprendizaje y aspectos sobre la estructura y el funcionamiento de las proteínas, partiendo de la necesidad de satisfacer las contradicciones que existan entre los dos aspectos antes señalados, según el modelo científico con cierto número de elementos y un "motor de inferencia" que los relaciona entre sí.

Considera hasta cuatro componentes del modelo relacionados con la forma cómo los alumnos elaboran sus conocimientos:

\section{Primer componente.-}

Para todo aprendizaje, lo identifica como el resultado de una actividad de elaboración que realiza el alumno movilizando sus concepciones ("estructuras de acogida") como preguntas, referentes, significaciones, operaciones, símbolos. Para tal fin, el sujeto utiliza estrategias para poder codificar las informaciones que recibe.

Las concepciones movilizadas facilitan que el estudiante relacione esas informaciones, sirven de "punto de anclaje" para generar nuevos significados: el sujeto elabora así su saber interactuando esas concepciones con las informaciones e interpretándolas.

No es frecuente que este proceso sea inmediato, es decir que no siempre es posible que muy pronto sean comprendidos los nuevos conocimientos. Sea porque el sujeto no disponga de la información necesaria; o porque aunque tenga accesibilidad no está motivado hacia ella, teniendo otra preocupación; o porque es incapaz de acceder a ella por limitaciones operativas, metodológicas, referenciales, entre otras; e incluso que carezca de los elementos adecuados para un desarrollo exitoso de la comprensión.

\section{Segundo componente.-}

En relación a los aprendizajes fundamentales, no es posible integrar automáticamente el nuevo conocimiento en la línea de los anteriores, los que representan, con frecuencia, un obstáculo para su integración.

Para tal fin, es necesario generar las condiciones suplementarias para el logro de la transformación radical de la malla conceptual, sugiriendo las siguientes pautas:

- El cuestionamiento constante por parte del alumno sobre sus concepciones, con el fin de superar el edificio establecido por el saber cotidiano y constituirlas en un "filtro" de la realidad. 
- El logro de la transformación de su concepción inicial, mediante la confrontación de ella a un conjunto de elementos convergentes y redundantes que la conviertan en contradicciones y difíciles de utilizar.

- La elaboración de una nueva malla conceptual mediante el uso de nuevas estrategias para conectar y relacionar las informaciones; para ello, requiere apoyarse en modelos organizadores que estructuren las informaciones de otro modo y faciliten respuestas correctas a las demandas que se presentan al alumno.

- Diferenciar progresivamente el campo de aplicación del concepto en elaboración, de su posterior movilización a situaciones diversas en las que llega a hacerlo operativo.

En consecuencia, el alumno trabaja contra esa concepción inicial pero partiendo de ella y con ella, hasta que llega el momento que la encuentra limitada, "mentirosa"o menos productiva que otras.

\section{Tercer componente.-}

Es necesario que el alumno ejerza un control deliberado en diferentes niveles, sobre los procesos que rigen el aprendizaje de conceptos.

El primer paso correspondería a que reorganice la información nueva, mediante su apreciación sobre cada situación, los significados que elabora y las representaciones del conocimiento.

Luego, debe conciliar el conjunto de parámetros anteriores, si es posible reutilizarlos, para elaborar un nuevo conocimiento.

Finalmente, procederá a la discriminación de las semejanzas y diferencias entre antiguos y nuevos conocimientos así como resolver las frecuentes contradicciones.

Se enfatiza en que los conceptos no se aprenden en corto tiempo y que el alumno necesitará informaciones complementarias o utilizar otros sistemas de relación; siendo necesario que previamente verifique que no le ha sido posible comprender ese concepto o que su sistema de pensamiento no es adecuado.

En general, comprenderá su estructura de conjunto cuando le es necesario examinarla sea para hacerla funcionar o para enseñarla.

\section{Cuarto componente.-}

Para garantizar el aprendizaje de conjunto deberá satisfacer las exigencias que sobre duración presentan los aprendizajes fundamentales, los que, como procesos múltiples, exigen tiempo para el logro exitoso de una serie de etapas sucesivas.

\section{El funcionamiento del modelo}

Se plantea ejecutar un proceso conflictivo en el que las adquisiciones anteriores proporcionarán el marco de cuestionamiento, referencial y de interpretación de la nueva adquisición de conocimientos de tipo conceptual; pero a la vez se sitúan en la ruptura con ellas, o en su modificación, o en su desviación. Cuando el alumno domina un nuevo modelo, produciéndose la comprensión de un fenómeno, se reorganiza el conjunto de su estructura mental y su aproximación a la realidad será diferente totalmente.

Giordán profundiza al respecto haciendo comparaciones entre el funcionamiento del pensamiento y del aprendizaje, con la estructura y funcionamiento de la enzima. Señala que de la misma forma que el saber se constituye a partir de las informaciones, las proteínas están constituidas por cadenas de aminoácidos unidos entre sí. Sin embargo, lo significativo en ambos casos son las diferentes relaciones establecidas entre partes de la cadena o entre distintas cadenas y no lo relacionado con el orden o sucesión de los elementos de esas cadenas. Las relaciones entre distintas cadenas son las constitutivas y corresponden a las que crean la estructura de la macromolécula, su fisonomía tanto interna como externa (Giordan, 1995).

Al carecer de importancia, para el aprendizaje del sujeto, el orden de registro de los datos, lo que se destaca es la pertinencia de la malla de relaciones establecidas entre su sistema conceptual y las informaciones registradas. Las que no podrán ser comprendidas, ni almacenadas, si no se interconectan significativamente con su marco de pensamiento. A ese nivel es posible definir "sitios activos conceptuales", por donde las nuevas informaciones podrían conectarse: sería como la base del proceso habitual de adquisición de datos que generalmente funciona al leer un diario o se mira un programa cultural en televisión, por ejemplo.

Puede ocurrir que las nuevas informaciones no pueden integrarse directamente sobre la existente estructura conceptual: pueden simplemente "pegarse" 
o ser eludidas o quedar aisladas. Razón por la que se hace indispensable deformar la estructura del pensamiento del sujeto $\mathrm{y}$, principalmente, cuando se trate de aprendizajes en profundidad, cobra importancia y significación la aplicación del modelo alostérico.

Giordán señala que es como si la estructura de la proteína pudiera totalmente modificarse si introdujéramos un nuevo aminoácido, un oligoelemento (transformación alostérica), de allí el nombre del modelo. Tal analogía es para describir cómo la estructura conceptual del alumno puede igualmente transformarse cuando nuevos elementos se introducen e integran en el conjunto. Constituyéndose así una nueva organización del saber con la posibilidad de hacerlo funcional y enriquecerlo.

\section{Significado del Modelo Alostérico para el Aprendizaje.}

A partir de lo planteado, se deduce la importancia de los esquemas de estructuración que existen entre los conceptos. Frente a un específico problema algunos conceptos cumplen el papel de organizadores y otros tienen roles secundarios: se plantea el problema de la integración de los conceptos en un conjunto que genera un significado particular que responde a ese problema específico.

Giordán refiere lo que ocurre con la proteína cuando privilegia relaciones funcionales con oligo-elementos o fosfolípidos en puntos muy precisos de su estructura, comparándolo con lo que ocurre con el alumno cuando aprende relacionando las informaciones exteriores, pero no de modo lineal, unas tras otras, como en la Pedagogía de la Transmisión descrita por Davini, C. (2001), sino relacionando esas informaciones con sitios característicos de su malla conceptual. Estos sitios facilitan la codificación de las informaciones así como la relación con la mismas y son activados por la situación de aprendizaje e igualmente son activados y transformados de modo prioritario para que se produzca la integración de los nuevos datos.

Con lo anterior se llega a elaborar un nuevo nivel de formulación del saber. Para los aprendizajes fundamentales, el nuevo dato no se agrega linealmente a los conocimientos anteriores, los que son, frecuentemente, un obstáculo para la integración de ese nuevo dato.
Para este caso, Giordan (1989) refiere que: “... el modelo alostérico propone elementos de comprensión: se observa, en efecto, modificaciones totales de la organización de ciertas proteínas bajo la influencia de un elemento suplementario. La secuencia de los aminoácidos permanece idéntica, pero se producen nuevas relaciones inter-cadenas, que provocan, en el caso de las enzimas, una modificación importante de su estructura y, por ello, de sus propiedades intrínsecas".

Además señala que para los aprendizajes conceptuales, es necesario el manejo de la deformación intelectual de los sitios activos de la estructura del pensamiento del estudiante que comparativamente con las proteínas, tal deformación específica puede generar una transformación radical de la malla conceptual. Es decir, llega a ser funcional otra estructuración del pensamiento, llegando los conceptos a tener importancia relativa, al ser conectados por otras relaciones que generan otros significados: las mismas informaciones permanecen pero no serán "leídas", ni discriminadas de la misma forma.

\section{El escenario del aprendizaje}

Siguiendo los postulados de Giordán, se puede plantear que el escenario del aprendizaje que toma en cuenta la posibilidad de las autotransformaciones, tiene dos componentes:

\section{1) Las condiciones del entorno didáctico}

La situación o el "entorno didáctico" requiere ser fuertemente favorecida para garantizar que el alumno, gracias a que dispone de un amplio sistema de interrelaciones en el "entorno didáctico", pueda hacer la actividad de elaboración, confrontando la información nueva y sus conocimientos movilizados y generando nuevas significaciones que respondan mejor a las interrogantes que se plantea.

Giordán destaca la importancia de presentar al alumno situaciones adecuadas, que cuestionen, con múltiples confrontaciones, pues aunque sea limitado el tiempo, sí podrá descubrir el conjunto de elementos necesarios para reorganizar las formulaciones, siempre y cuando cuente con el acceso a cierto número de elementos significativos como documentos, experimentos, argumentos y de formalismos mínimos como gráficos, simbolismos, esquemas, modelos. Bajo estas condiciones podrá confrontar lo aprendido con la nueva información por aprender. 
El autor refiere: "Es preciso, pues, abandonar la idea de dejar a los alumnos que elaboren los conceptos a partir de la observación o la experiencia de clase exclusivamente. Ello no significa que sea necesario volver indefectiblemente a un proceso expositivo tradicional; somos conscientes de que "dar" o "mostrar" una noción no es algo operatorio, salvo excepciones en las que la estructura de pensamiento está lista para aceptar directamente la nueva información, lo que es acorde con el modelo alostérico expuesto".

Considera que los parámetros significativos que deben estar presentes en el entorno didáctico están relacionados con los siguientes mecanismos de autotransformación:

\section{a) Introducir los desequilibrios conceptuales pertinentes}

Los desequilibrios conceptuales pertinentes se introducen para que el alumno se oriente a una actividad constructiva; y para tal fin, es indispensable motivarlo en cuanto al tema a tratar, que tenga un cierto nivel de actitud, de dominio de los procesos científicos y con capacidad para explicitar su pensamiento y de ponerlo a prueba.

También es indispensable la confrontación auténtica de sus ideas con la realidad, con otro alumno, con la información, con el maestro; para que se convenza de la inadecuación de sus concepciones en relación con el problema tratado.

Por ello es necesario que se confronte con diferentes argumentos para que relacione un conjunto de nuevos datos que le inducirán a relativizar el valor de sus evidencias anteriores, a reformular el problema $\mathrm{y} / \mathrm{o}$ enfrentar otras relaciones.

\section{b) Acceso al formalismo}

El alumno accederá a símbolos, esquemas, modelos de fácil uso, para que organice los nuevos datos y le sirvan de punto de anclaje para producir la nueva estructura del conocimiento; con ello tendrá mayor facilidad para relacionar lo conocido con lo nuevo, para actualizar la información conocida, o para discriminar el común denominador de un conjunto de fenómenos comunes.

Para evitar que los nuevos datos percibidos permanezcan aislados, el alumno utilizará el formalismo para romper con el marco organizador antiguo y producir nuevos significados.
En consecuencia, al inducir nuevos modelos tendrá una renovada visión de la realidad, el alumno contará con un "núcleo resistente" para relacionar datos y producir nuevos pensamientos.

Es aconsejable que, en una primera etapa, el profesor proporcione un esbozo de modelo o pre modelo, elaborado de modo comprensible, legible y adaptado a la percepción del alumno acerca del problema, con el propósito inmediato de familiarizarlo con su uso, con la posibilidad de que lo haga funcionar y produzca nuevas ideas con él.

Al conocer el profesor las actividades de elaboración necesarias para que el estudiante logre un aprendizaje conceptual, podrá orientarlo hacia la ejecución de la actividad que falta y ayudarlo por medio de procedimientos didácticos adecuados que le faciliten actualizar concepciones o actividades vinculadas a ellas.

\section{2) Otras condiciones complementarias}

Finalmente, el autor plantea las condiciones que pueden complementar a los parámetros anteriores, destacando las siguientes:

\section{a) Movilizar el nuevo conocimiento para comprobar su operatividad y limitaciones}

Esta condición mostrará al estudiante que los nuevos datos son más fácilmente aprendidos cuando éstos se integran en estructuras y lo habituará a insertar lo nuevo en lo anterior, situándose entre lo nuevo por aprendery lo ya aprendido, activando los conocimientos anteriores necesarios e interrelacionándolos utilizando sus mecanismos cognitivos.

\section{b) Desarrollo de un conocimiento sobre el conocimiento}

Mediante la reflexión acerca de las prácticas conceptuales para conocer el aporte e interés sobre ellas, así como discriminar las "lógicas" subyacentes en el desarrollo de los procesos.

Esta condición se basa en que no siempre los obstáculos en el aprendizaje se ligan directamente al saber en sí mismo, sino que dependen de la imagen del alumno o de su epistemología intuitiva sobre el proceso experimental o sobre sus mecanismos de adquisición del saber. 


\section{c) Papel primordial e irremplazable del profesor}

Ejerciendo su función de organizador de las condiciones del aprendizaje, el enseñante ofrece al aprendiz el entorno didáctico mediante aportaciones, interacciones y situaciones facilitadoras, que propicien el funcionamiento de sus conocimientos científicos y técnicos, sin olvidar que es el alumno el que elabora, relaciona, integra; es decir, aprende en base de sus propias estructuras de pensamiento.

\section{APROXIMACIÓN DEL MODELO ALOSTÉRICO DE APRENDIZAJE A LA CAPACITACIÓN DE ADULTOS}

Las actividades de capacitación de adultos, habitualmente, se desarrollan siguiendo un enfoque acumulativo, inorgánico, no estructurado. Empleando una analogía, postulamos que la capacitación se planifica como quien traza una urbanización en un terreno eriazo y se limita a acumular los materiales en zonas pre fijadas para que alguien levante un edificio pensado como una vivienda, una fábrica o un templo; pero los cimientos ya existen y cuando no son adecuados, la edificación presenta fallas que nos impiden continuar construyendo.

Este modelo, el tradicional, es inapropiado en la capacitación de adultos, quienes traen consigo experiencias, conceptos, modelos, valores, motivaciones, prejuicios y resistencias. Según esto, la limitación tradicional de la capacitación de adultos empieza con una notable desventaja de inicio: supone que va a construir sobre un terreno baldío, cuando ya existen en él edificaciones de distinta altura y solidez.

La transferencia indebida de enfoques pedagógicos para niños, cuyo entendimiento es como una pizarra limpia, hacia adultos; es decir, la reducción de la andragogía a la pedagogía, ha significado una frustración para transformar el conocimiento, entendimiento y comprensión de los adultos.

Las evaluaciones convencionales orientadas a verificar la posesión de información, especialmente con pruebas como la de elección múltiple, no garantizan la aplicación de la misma a las rutinas comunicativas de la práctica profesional.

Tal limitación ha generado que en los últimos años no sólo se considere el análisis cuantitativo tradicional de los resultados del aprendizaje de un grupo de estudiantes, sea cual fuere su escolaridad y nivel.
Es decir que, adicionalmente, en toda evaluación se incorpora el análisis cualitativo, cobrando éste mayor relevancia y utilidad que el cuantitativo, principalmente cuando se trata de discriminar las fallas en los procesos educativos y de capacitación.

En consecuencia, considerando al modelo alostérico de aprendizaje de Giordán como una innovación para el análisis cualitativo del aprendizaje en niños; $y$, tratando de incidir en el mapa cognitivo que tiene el adulto, en reparar las estructuras que deforman su conocimiento y comprensión y en modificar su posición afectivo-valorativa respecto a un tema, es posible su aplicación al campo de la Capacitación de Adultos.

Comparativamente, en el enfoque tradicional, los objetivos identifican el conocimiento que debe ser entregado. En el enfoque alostérico, los objetivos identifican prioritariamente las resistencias y el conocimiento previo y antagónico que bloquea la incorporación de nueva información relevante, aunque aparentemente sea comprendida y aprendida.

El primer paso del enfoque es la adecuación del edificio de conocimientos previamente existente, cuando menos en sus estructuras más importantes. En este sentido, la evaluación inicial no se interesa únicamente en estimar el nivel de conocimientos de entrada. La evaluación inicial, que es capital, busca identificar los conocimientos e ideas contrapuestos al nuevo conocimiento materia de la capacitación, sus raíces y los medios para erradicarlos. En pocas palabras, la evaluación inicial debe identificar los errores conceptuales y su trama de conocimientos de otra índole.

Como vivimos en la era de la información y del conocimiento, el esfuerzo didáctico en la capacitación no sólo tiene que aportar información organizada para construir conocimiento. Esta entrega ocurre en cada momento y por varias vías, por los medios de comunicación disponibles; $y$, este esfuerzo tiene que identificar el conocimiento que opera como resistencia o como información. Si avanzamos en este camino, la transformación de los adultos, en esta época de cambios intensos, podrá ponerse a tono con los tiempos.

La capacitación de adultos, entendida como una modalidad condicionada por factores de cambio en la vida política, económica, social y cultural de un país, está dirigida a satisfacer esas demandas en los niveles educativos posteriores o diferentes a la educación 
formal del adulto, sea en la comunidad, en el hogar o en su lugar de trabajo.

Dado que es muy útil la aproximación del Modelo Alostérico de Aprendizaje de André Giordán a la capacitación de adultos, también lo es en el campo de la Salud Pública. Por ejemplo, en temas relacionados con la salud de los escolares, el ejercicio profesional de los profesores tiene un efecto multiplicador en la medida que transmite conocimientos a decenas de estudiantes, pero también tiene un efecto amplificador, de concepciones correctas y erróneas, en la medida que se le considere fuente de información válida. Por ello, se hace necesario planificar para los profesores Programas de Capacitación que tengan en cuenta criterios más estrictos tanto para su diseño, como para su evaluación. Es decir, las consecuencias negativas del efecto amplificador y del efecto multiplicador de los errores y distorsiones de sus profesores, en la educación de niños y adolescentes, es enorme comparándola con la consecuencia positiva de los aprendizajes correctos (Giordan, 1995).

En consecuencia, no será suficiente considerar alcanzadas las metas y objetivos de los Programas de Capacitación en función del mayor número de profesores asistentes, basados únicamente en evaluaciones meramente cuantitativas, que no garantizan beneficios de los aparentes logros, corriéndose el riesgo de que en las aulas la información que se esté dando a los alumnos tenga distorsiones perjudiciales para su educación. No será suficiente entonces continuar haciendo la evaluación del aprendizaje en base del puntaje de respuestas correctas que alcance el capacitado.

Cuando se trata de temas como la Salud Sexual y Reproductiva sobre los que un profesor debe tener excelente capacitación, se requiere un aprendizaje cien por ciento correcto. De lo contrario, al transmitirse los errores, influirá negativamente en el manejo de los problemas característicos de la adolescencia como: el embarazo precoz, el aborto, y las enfermedades transmisibles sexualmente, entre otros.

Considerando lo planteado por el Modelo Alostérico de Aprendizaje que prioriza la relación entre una estructura de pensamiento ya constituida con los nuevos conocimientos, se intenta destacar que, comparativamente con los niños, en los adultos esta relación requiere aún más de un análisis cuidadoso que considere la diversidad de conceptos que, a lo largo de su vida y en diferentes contextos, el adulto ha ido aprendiendo, y no siempre de modo correcto.
Se debe profundizar más en el análisis de sus errores, sugiriendo que se inicie con la cuantificación de tal evaluación teniendo en cuenta las cuatro opciones denominadas como DOBA: Distorsiones, Omisiones, Buenas y Aciertos, Modelo creado por González, D. (1998); para luego recoger información cualitativa de las distorsiones y omisiones, destacando para el grupo de estudio y, en general para los adultos, la relacionada con las fuentes del aprendizaje y su interpretación.

El uso del DOBA constituye un pre-requisito para aplicar el modelo alostérico, una alternativa innovadora en cuanto a evaluación del aprendizaje, la base objetiva para el análisis cualitativo de la aplicación de tal modelo y garantiza la discriminación de las concepciones erróneas en los alumnos.

Otro elemento también importante es el análisis posterior mediante la entrevista a profundidad que se requiere utilizar para discriminar las características peculiares de los conceptos distorsionados, a la que se agrega el uso de una guía sugiriendo pautas para generar mecanismos de autotransformación que permitan cambiar esas distorsiones en conceptos correctos.

La aproximación del modelo alostérico al campo de la Capacitación de Adultos es amplia y compleja; más aún, tratándose de elementos relacionados con el campo cognitivo del ser humano que cubre diversidad de aspectos como ideas, conceptos, principios, definiciones, abstracciones, deducciones; incluyendo, además, diversidad de mecanismos, estilos cognitivos y mapas cognitivos, de los que hace uso para su funcionamiento y desarrollo.

Eligiendo, en esta amplitud, el interés de tal aproximación se orienta a la discriminación -dentro del campo cognitivo- de los errores (distorsiones y omisiones) de los adultos, así como a los mecanismos que propicien su autotransformación.

El esfuerzo representa una alternativa muy concreta que plantea conocer detalladamente las características singulares de las concepciones erróneas para que las modifique, dado que al transformarlas en correctas, éstas se constituirán en adecuados puntos de anclaje para los nuevos aprendizajes.

En todo proceso de enseñanza-aprendizaje las ideas previas no deben ser ignoradas, sino que deben tomarse en cuenta como base para la adquisición de los nuevos conceptos. 
Reconocida la amplitud y complejidad del campo cognitivo, el interés central se ubica en caracterizar en el grupo de adultos, una vez discriminadas sus distorsiones más frecuentes, dos aspectos necesarios e importantes para que en el futuro les garanticen el logro de aprendizajes correctos para ellos. Un aspecto es el relacionado con las condiciones que todo sujeto debe poner en marcha para lograr la autotransformación de su distorsión. El otro aspecto está referido a darle apoyo para que tenga el entorno didáctico idóneo que favorezca ese cambio.

El análisis de la concepción distorsionada requiere profundizar en no sólo los componentes sugeridos por el modelo alostérico, sino que además, por su condición de adulto, se hace necesario conocer la historia previa de su "malla conceptual" constituida no sólo por capacitaciones, sino principalmente por sus experiencias de vida y por otras fuentes de aprendizaje que no necesariamente corresponden a las de la enseñanza formal.

Es por ello que considerando lo planteado por André Giordán en su modelo, la aproximación al campo de los adultos requiere agregar otros componentes como el afectivo y el actitudinal, que agregados al factor experiencia, deben estar siempre presentes en el análisis.

En cuanto a la participación del investigador para optimizar el entorno didáctico que facilite en cada profesor las autotransformaciones de sus distorsiones, esta intervención ha sido no sólo posible, sino muy ágil. Gracias a la experiencia y preparación pedagógica que los docentes poseen en este campo, fue posible manejar con mucha facilidad las confrontaciones entre conceptos así como el uso de símbolos, esquemas y modelos para reorganizar la información y generar las concepciones correctas sobre los temas.

Este segundo aspecto, a diferencia del trabajo con niños, en el campo de la Capacitación de Adultos es muy probable que para determinadas profesiones se logre de modo exitoso. Razón por la que los mecanismos de autotransformación para el caso de los adultos no requerirá tanto del factor tiempo, como plantea el autor para los niños, sino de las destrezas y habilidades cognitivas del adulto para el uso de sus formalismos y su lógica.

Es decir, aunque el autor plantea que en los niños no siempre es posible que este proceso sea inmediato, en el caso de los adultos las condiciones se presentan de modo distinto debido, principalmente, a su experiencia para movilizar sus concepciones, plantearse contradicciones, dar nuevos significados, utilizar otros símbolos y operaciones. De este modo, los adultos utilizan estrategias cognitivas que les permitan codificar adecuadamente la información y transformar la distorsión.

Sin embargo, en la aproximación del modelo a la capacitación de los adultos sí se conserva la analogía entre los procesos de aprendizaje y los aspectos sobre la estructura y funcionamiento de las proteínas, partiendo de la necesidad de satisfacer las contradicciones que existan al interior de cada aprendizaje. En tal sentido, es muy útil tal analogía para entender la elaboración de la malla conceptual y cómo las distorsiones impiden que los nuevos aprendizajes se asimilen correctamente a ella, quedando éstos aislados y dispersos al no encajar adecuadamente entre sí.

Dado que tales concepciones constituyen el punto de anclaje para los nuevos aprendizajes, al no ser correctas, es sumamente importante discriminarlas para su posterior autotransformación y adecuación a la malla conceptual y evitar nuevas distorsiones.

Al confrontar el concepto con otros elementos, el sujeto no lo encuentra coherente, ni lógico y se interesa por conocer el concepto correcto, la razón de tal respuesta, procesa su lógica y coherencia y autotransforma su concepción en base de las contradicciones.

Se reitera lo comentado en la descripción del modelo alostérico: el alumno trabaja contra esa concepción inicial pero partiendo de ella y con ella, hasta que llega el momento que la encuentra limitada, "mentirosa" o menos productiva que otras, autotransformándola en una concepción correcta.

Reconocida la complejidad del campo cognitivo del ser humano, cuyo estudio todavía es considerado como inacabable, y analizada la amplitud de acciones a las que puede aplicarse el modelo alostérico de aprendizaje, esta aproximación al campo de la Capacitación de Adultos como tal, es el primer paso, es un primer esfuerzo centrado exclusivamente en discriminar distorsiones para luego autotransformarlas en profesores de educación secundaria capacitados en Educación Sexual.

La aplicación del modelo va más allá de la autotransformación de las distorsiones, éstas sólo constituyen un pre-requisito, pero de suma importancia, para la adquisición de nuevos aprendizajes que sean 
los fundamentales o los conceptuales y no se puede dejar de reconocer que ambos aprendizajes a su vez constituyen el pilar, la base esencial de la producción creativa del sujeto frente a las demandas del mundo exterior.

La cadena se asegura empezando por corregir las distorsiones de los conceptos existentes y es posible continuar investigando y abordando lo relacionado con los nuevos aprendizajes e ir avanzando hasta generar las óptimas condiciones para desarrollar la creatividad en el ser humano.

Otra posible razón, estaría relacionada de modo significativo también con las distorsiones de sus conceptos pues al no tenerlos incorporados correctamente a su malla conceptual, los datos que posee el profesor estarían generando a la vez opiniones y prácticas inapropiadas e incoherentes entre sí.

La inquietud por la innovación pedagógica encontró en el modelo alostérico una nueva respuesta, un reto complejo por sus elementos cognitivos que, no se pretende aislarlos, sino integrarlos al conjunto; y, permite plantear la Pedagogía de la Autotransformación como alternativa innovadora frente a propuestas unilineales como la Pedagogía clásica de la Transmisión frecuentemente desplazada por la Pedagogía del Adiestramiento y la Pedagogía de la Problematización. Pedagogías de amplio uso en la Capacitación de Adultos, carentes del análisis profundo de las cogniciones del sujeto que se capacita y se orientan al uso de metodologías consideradas ahora como tradicionales.

Con mayor razón entonces es necesario romper con los esquemas tradicionales de evaluación del proceso enseñanza-aprendizaje, pues partiendo del análisis de los errores en el aprendizaje e interviniendo el propio aprendiz para la autocorrección de sus distorsiones, será posible garantizar la optimización de los logros de los objetivos y las metas del proceso y disminuir la probabilidad de incoherencias entre sus opiniones y sus prácticas.

El presente artículo ha sido adaptado de la Tesis de Doctorado de la autora. Aguilar, A. (1997). Una aproximación del modelo alostérico de aprendizaje a la capacitación de adultos: análisis cualitativo de las concepciones distorsionadas sobre educación sexual de profesores de educación secundaria. Tesis para optar el grado de Doctor en Salud Pública. Universidad Peruana Cayetano Heredia.

\section{REFERENCIAS BIBLIOGRÁFICAS}

1. Aguilar, A. (1997). Una aproximación del modelo alostérico de aprendizaje a la capacitación de adultos: análisis cualitativo de las concepciones distorsionadas sobre educación sexual de profesores de educación secundaria. Tesis para optar el grado de Doctor en Salud Pública. Universidad Peruana Cayetano Heredia. Lima, Perú. 1997.

2. Ausubel, D. (1982). Psicología Educativa. Un punto de vista cognoscitivo. México DF: Trillas.

3. Bandura, A. (1983). Principios de la modificación de conducta. Salamanca: Sígueme.

4. Davini, C. (2001). Modelos y opciones pedagógicas. (Citado en enero del 2007) Disponible en: http:// www.fisicanet.com.ar/docentes/curso/ apuntes/27_04_1.php

5. Gagné, R. (1979). Las condiciones del aprendizaje. México: Nueva Editorial Interamericana.

6. Giordan, A. (1989). Representaciones sobre la utilización didáctica de las representaciones Enseñanza de las ciencias. Revista de investigación y experiencias didácticas, 7(1), 53-62

7. Giordan, A. (1995). Autobiography. (Citado en enero del 2010) URL Disponible en: http://www. andregiordan.com/englishbio.html

8. Giordan, A. (1995). Los nuevos modelos de aprendizaje: ¿más allá del constructivismo? Perspectivas, 1, 107124.

9. González, D. (1998) El Modelo DOBA para la evaluación de conceptos: aspectos prácticos. III Simposio Filosofía de la Medicina. Escuela de Postgrado Víctor Alzamora Castro, Universidad Peruana Cayetano Heredia. Lima, Perú.

10. Harper, H (1994) Tratado de bioquímica. México: Manual Moderno.

11. Lehninger, A. (1993). Principios de bioquímica. Barcelona: Omega.

12. Monod, J., Wyman, J., \& Chageux, J. (1995). On the nature of allosteric transitions: a plausible model. J Molec Viol, 12,88-118.

13. Monod, J. (1993) El azar y la necesidad. Ensayo sobre la Filosofía Natural de la Biología Moderna. Madrid, España: Planeta Agostini.

14. Skinner, B.F. (1979) La conducta de los organismos: un análisis experimental. Barcelona: Fontanella.

15. Skinner, B. F. (1979) Contingencias de reforzamiento: un análisis teórico. México DF: Trillas. 